\title{
Longitudinal Dietary Trajectories from Preconception to Mid-Childhood in Women and Children from the Southampton Women's Survey: A Group Based Trajectory Modelling Approach
}

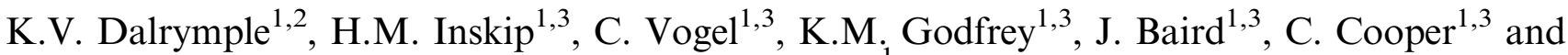 \\ S.R. Crozier ${ }^{1}$ \\ ${ }^{1}$ MRC Lifecourse Epidemiology Unit, Southampton General Hospital, University of Southampton, Southampton, UK, \\ ${ }^{2}$ Department of Women and Children's Health, School of Life Course Sciences, King's College London, London, UK \\ and \\ ${ }^{3}$ NIHR Southampton Biomedical Research Centre, University of Southampton and University Hospital Southampton \\ NHS Foundation Trust, Southampton, UK
}

The latest figures from the National Child Measurement Programme in England indicate that nearly $25 \%$ of 4-5 year-old children are overweight or obese. This rises to a third by the time they leave primary school ${ }^{(1)}$. Diet quality in early life, even prior to conception ${ }^{(2)}$, is increasingly considered to be an important modifiable factor contributing to the development of obesity ${ }^{(3)}$. Evaluation of longitudinal mother/offspring diet quality trajectories from before pregnancy through early life may enable identification of subgroups most at risk of poor diet quality, and optimal timepoints for interventions to help prevent childhood obesity.

2936 women and their children from the Southampton Women's Survey were included in this analysis. Using intervieweradministered food frequency questionnaires, maternal diet was assessed pre-pregnancy, at 11 and 34 weeks' gestation, and offspring diet at ages 6 months, 12 months, 3, 6-7 and 8-9 years. At each time point a standardised dietary quality index was derived using principal component analysis. Standardised adiposity outcomes were collected at $8-9$ years of age and included dual-energy X-ray absorptiometry body fat percentage, WHO BMI z-scores and circumferences. We aimed to [1] define latent groups of dietary trajectories from pre-pregnancy to 8-9 years of age using group-based trajectory modelling of the dietary quality index, [2] examine associations between early life sociodemographic variables and these trajectories and [3] describe the association between the trajectories and adiposity outcomes at age $8-9$ years $(n=1216 / 2963)$.

A 5-trajectory group model was identified as the optimal model. The diet quality trajectories were characterised as stable, horizontal lines, and were defined as low $(n=142)$, low-medium $(n=655)$, medium $(n=1146)$, medium-high $(n=818)$ and high $(n=163)$. Higher maternal pre-pregnancy BMI, smoking and multiparous mothers, lower maternal age at birth and lower education attainment, and lower offspring birthweight and shorter breastfeeding duration were associated with dietary trajectories of lower quality. There were strong associations between lower diet quality trajectories and higher adiposity at age $8-9$ years. These findings were robust following adjustment for DAG-derived confounders: pre-pregnancy BMI, maternal highest education attainment and age at birth. A one-class decrease in the diet quality trajectory was associated with a higher percentage body fat $(0.09 \mathrm{SD}(95 \%$ confidence interval $0.01,0.17)$, BMI z-score $(0.09 \mathrm{SD}(0.015,0.17)$ and waist circumference $(0.07 \mathrm{SD}(0.005,0.13)$.

This study has identified that diet quality trajectories are stable from preconception into childhood, and a lower trajectory is associated with maternal socio-demographic factors, age and parity, and with adiposity in the child at age 8-9-years. These findings suggest that targeted intervention strategies during the preconception period may be an opportune window to promote positive dietary changes in the mother and subsequently the child, therefore reducing the risk of the child developing obesity in early life.

\section{References}

1. Public Health England, NHS National Child Measurement Programme: 2019/20 School Year.

2. Stephenson J, Heslehurst N, Hall J, et al. (2018) Lancet 391 (10132), 1830-1841.

3. Rolland-Cachera MF, Akrout M \& Péneau S (2016) Int J Environ Res Public Health 13, 564. 